\author{
K. RAUF, B. Y. AIYETAN, D. J. RAJI AND R. U. KANU \\ (Received 19 April 2017; Revision Accepted 21 July 2017)
}

\begin{abstract}
In this paper, some fixed point theorems were proved, to show the existence and uniqueness of a fixed point under some weaker contractive conditions in a complete G-metric space settings. Moreover, we obtain the G-Cauchy sequence for the unique fixed point. Our results extend and refine some recent results in the literature.
\end{abstract}

KEYWORDS AND PHRASES: G-metric, G- Cauchy, G- limit, G-continuous and weak contractive conditions.

\section{INTRODUCTION}

Most of the problems that occur in life are nonlinear in nature but fixed point theory depends on the linear structure of normed linear spaces or Banach spaces setting. However, a nonlinear framework for fixed point theory is a metric space embedded with a structure.

In 2017, Rauf et.al. [10] introduced some new implicit Kirk-type iterative schemes in generalized convex metric spaces in order to approximate fixed points for general class of quasicontractive type operators. The strong convergence, T-stability, equivalency, data dependence and convergence rate of these results were explored. Their iterative schemes are faster and better, in term of speed of convergence, than their corresponding results in the literature. The results also improved and generalized several existing iterative schemes in the literature and they provided analogues of the corresponding results of other spaces, namely: normed spaces, CAT(0) spaces and so on.

Mustafa and Sim in 2006, [8] Introduced a new notion of generalized metric space called G-metric space, after proving that most of the result concerning the topological properties of D-metric space were incorrect. To repair this setback, they gave a more appropriate notion of a generalized metrics, called Gmetric space. For more details on G-metric space, see $\left[\begin{array}{lll}6 & \& & 9\end{array}\right]$ and the reference therein.

\title{
2. PRELIMINARIES AND DEFINITIONS
}

In this section, we recollect some basic definitions and overview of the fundamental results.

Definition 1 (Mustafa and Sim [8]): A G-metric space is a pair $(X, G)$ where $X$ is a nonempty set and $G: X \times X \times$ $X \rightarrow[0, \infty)$ is a function such that, for all $x, y, z, a \in X$, the following conditions are fulfilled:

(G1) $\quad G(x, y, z)=0$ if $x=y=z$

(G2) $G(x, x, y)>0$ for all $x, y \in X$ with $x \neq y$;

(G3)

$G(x, x, y)>0$ for all $x, y \in X$ with $x \neq y$;

(G4) $\quad G(x, y, z)=G(x, z, y)=G(y, z, x)$

(G5) $\quad G(x, y, z) \leq G(x, a, a)+G(a, y, z)$

In such a case, the function $G$ is called a G-metric on $X$.

K. Rauf, Department of Mathematics, University of Ilorin, Ilorin, Kwara State, Nigeria.

B. Y. Aiyetan, Department of Mathematics, University of Ilorin, Ilorin, Kwara State, Nigeria.

D. J. Raji, Department of Mathematics, University of Ilorin, llorin, Kwara State, Nigeria.

R. U. Kanu, Department of Basic Sciences (Mathematics Unit), Babcock University, Ilishan- Remo, Ogun State, Nigeria. 
Lemma 2.1 (Mustafa and Sim [8]): Let $(X, G)$ be a G-metric space. Then, for any $x, y, z, a \in X$, the following properties hold:

1. $G(x, y, z) \leq G(x, x, y)+G(x, x, z)$;

2. $\quad G(x, y, z) \leq G(x, a, a)+G(y, a, a)+G(z, a, a)$;

3. $|G(x, y, z)-G(x, y, a)| \leq \max \{G(a, z, z), G(z, a, a)\}$;

4. If $n \geq 2$ and $x_{1}, x_{2} \ldots x_{n} \in X$, then

and

$$
G\left(x_{1}, x_{n}, x_{n}\right) \leq \sum_{i=1}^{n-1} G\left(x_{i}, x_{i+1}, x_{i+1}\right)
$$

$$
G\left(x_{1}, x_{1}, x_{n}\right) \leq \sum_{i=1}^{n-1} G\left(x_{i}, x_{i}, x_{i+1}\right)
$$

5. If $G(x, y, z)=0$, then $x=y=z$;

6. $\quad G(x, y, z) \leq G(x, a, z)+G(a, y, z)$;

7. $\quad G(x, y, z) \leq \frac{2}{3}[G(x, y, a)+G(x, a, z)+G(a, y, z)]$

8. If $x \in X\{z, a\}$, then $|G(x, y, z)-G(x, y, a)| \leq G(a, x, z)$; and

9. $\quad G(x, y, y) \leq 2 G(x, y, z)$.

Definition 2.2: (Agarwal et.al. [1]): Let $(X, G)$ be a G-metric space, let $x \in X$ be a point and let $\left\{x_{n}\right\} \subseteq X$ be a sequence. We say that:

1. $\left\{x_{n}\right\}$ G-converges to $x$, and we write $\left\{x_{n}\right\} \rightarrow x$, if $\lim _{n, m \rightarrow \infty} G\left(x_{n}, x_{m}, x\right)=0$, that is, for all $\varepsilon>0$ there exists $n_{0} \in \mathbb{N}$ satisfying $G\left(x_{n}, x_{m}, x\right) \leq \varepsilon$ for all $n, m \in \mathbb{N}$ such that $n, m \geq n_{0}$ (in such case, $x$ is the G-limit of $\left\{x_{n}\right\}$;

2. $\left\{x_{n}\right\}$ is G-Cauchy if $\lim _{n, m \rightarrow \infty} G\left(x_{n}, x_{m}, x_{k}\right)=0$, that is, for all $\varepsilon>0$ there exists $n_{0} \in \mathbb{N}$ satisfying $G\left(x_{n}, x_{m}, x_{k}\right) \leq \varepsilon$ for all $n, m, k \in \mathbb{N}$ such that $n, m, k \geq n_{0}$;

3. $(X, G)$ is complete if every G-Cauchy sequence in $X$ is G-convergent in $X$.

Definition 2.3: (Agarwal et.al. [1]): Let $(X, G)$ be a G-metric space, let $\left\{x_{n}\right\} \subseteq X$ be a sequence and let $x \in X$. Then the following conditions are equivalent.

1. $\left\{x_{n}\right\}$ G-convergents to $\mathrm{x}$;

2. $\lim _{n, m \rightarrow \infty} G\left(x_{n}, x_{m}, x_{m}\right)=0$;

3. $\lim _{n, m \rightarrow \infty, m \geq n} G\left(x_{n}, x_{m}, x_{n}\right)=0$;

4. $\quad \lim _{n, m \rightarrow \infty, m>n} G\left(x_{n}, x_{m}, x_{m}\right)=0$;

5. $\lim _{n, m \rightarrow \infty} G\left(x_{n}, x_{n}, x_{m}\right)=0$;

6. $\lim _{n, m \rightarrow \infty, m \geq n} G\left(x_{n}, x_{n}, x_{m}\right)=0$;

7. $\lim _{n, m \rightarrow \infty, m>n} G\left(x_{n}, x_{n}, x_{m}\right)=0$;

8. $\lim _{n \rightarrow \infty} G\left(x_{n}, x_{n+1}, x_{n+1}\right)=0$ and $\lim _{n, m \rightarrow \infty, m>n} G\left(x_{n}, x_{n+1}, x_{m}\right)=0$.

Definition 2.3: (Agarwal et.al. [1]): Let $(X, G)$ be a G-metric space. We say that:

a. A mapping $T: X \rightarrow X$ is G-continuous at $x \in X$ if $\left\{T x_{m}\right\} \rightarrow T x$ for all sequence $\left\{x_{n}\right\} \subseteq X$ such that $\left\{x_{m}\right\} \rightarrow x$;

b. $\quad$ A mapping $F: X^{n} \rightarrow X$ is $\mathrm{G}$-continuous at $\left(x_{1}, x_{2}, \ldots, x_{n}\right) \in X^{n}$ if

$$
\left\{F\left(x_{1}^{m}, x_{2}^{m}, \ldots, x_{n}^{m}\right) \rightarrow F\left(x_{1}, x_{2}, \ldots, x_{n}\right)\right.
$$

For all sequence $\left\{\left(x_{1}^{m}, x_{2}^{m}, \ldots, x_{n}^{m}\right)\right\} \subseteq X^{n}$ such that $\left\{x_{i}^{m}\right\} \rightarrow x_{i}$ for all $i \in\{1,2, \ldots, n\}$;

c. a mapping $H: X^{n} \rightarrow X^{m}$ is G-continuous at $\left(x_{1}, x_{2}, \ldots, x_{n}\right) \in X^{n}$ if $\pi_{i}^{m} \circ H: X^{n} \rightarrow X$ is G-continuous at $\left(x_{1}, x_{2}, \ldots, x_{n}\right)$ for all $i \in\{1,2, \ldots, m\}$, where $\pi_{i}^{m}: X^{m} \rightarrow X$ is the ith-projection of $X^{m}$ onto $X$ (that is, $\pi_{i}^{m}\left(a_{1}, a_{2}, \ldots, a_{m}\right)=a_{i}$ for all $\left.\left(a_{1}, a_{2}, \ldots, a_{m}\right) \in X^{m}\right)$.

Theorem 2.1: (Agarwal et.al. [1]): If $(X, G)$ be a $\mathrm{G}$ metric space. Then the function $G(x, y, z)$ is jointly continuous in all three of its variables, that is, if $x, y, z \in X$ and $\left\{x_{n}\right\},\left\{y_{n}\right\},\left\{z_{n}\right\} \subseteq X$ are sequences in $X$ such that $\left\{x_{n}\right\} \stackrel{G}{\rightarrow} x,\left\{y_{n}\right\} \stackrel{G}{\rightarrow} y$ and $\left\{z_{n}\right\} \stackrel{G}{\rightarrow} z$, then $\left\{G\left(x_{m}, y_{m}, z_{m}\right)\right\} \rightarrow G(x, y, z)$. 
3. MAIN RESULT

Here, we study some results related to unique fixed point $p$, and show that mapping $T$ is G- continuous at $p$. We shall establish the results in sequel as follows.

Theorem 3.1: Let $(X, G)$ be a complete G-metric space and let $T: X \rightarrow X$ be a mapping satisfying the following conditions:

$$
\begin{aligned}
& G(T x, T y, T z) \leq \alpha G(x, T x, T x)+\varphi G(x, y, z) \\
& G(T x, T y, T z) \leq \alpha G(x, x, T x)+\varphi G(x, y, z)
\end{aligned}
$$

For all $x, y, z \in X$ where $0 \leq \alpha+\varphi<1$, then $T$ has a unique fixed point $p$ in $T ; T p=p$.

Proof: Let $\alpha$ and $\varphi$ be a contraction constant of the mapping $T$; let $x_{0}$ be an arbitrary but fixed element in $X$. Define the sequence of iterate $\left\{x_{n}\right\}$ in $X$ as

$$
x_{n}=T^{n} x_{0} \quad \text { For all } n \geq 1
$$

If $x_{n+1}=y_{n}=z_{n}$ and since $T$ is a contraction satisfying (3.1) or (3.2) we have

$$
\begin{gathered}
G\left(x_{n}, x_{n+1}, x_{n+1}\right)=G\left(T x_{n-1}, T x_{n}, T x_{n}\right) \\
\leq \alpha G\left(x_{n-1}, x_{n}, x_{n}\right)+\varphi G\left(x_{n}, x_{n+1}, x_{n+1}\right) \\
=\alpha G\left(T x_{n-2}, T x_{n-1}, T x_{n-1}\right)+\varphi G\left(T x_{n-1}, T x_{n}, T x_{n}\right) \\
\leq \alpha^{2} G\left(x_{n-2}, x_{n-1}, x_{n-1}\right)+\varphi^{2} G\left(x_{n-1}, x_{n}, x_{n}\right) \\
=\alpha^{2} G\left(T x_{n-3}, T x_{n-2}, T x_{n-2}\right)+\varphi^{2} G\left(T x_{n-2}, T x_{n-1}, T x_{n-1}\right) \\
\leq \alpha^{3} G\left(x_{n-3}, x_{n-2}, x_{n-2}\right)+\varphi^{3} G\left(x_{n-2}, x_{n-1}, x_{n-1}\right)
\end{gathered}
$$

Continue iteratively leads to

$$
\leq \alpha^{n} G\left(x_{0}, x_{1}, x_{1}\right)+\varphi^{n} G\left(x_{1}, x_{2}, x_{2}\right)
$$

Hence

$$
G\left(x_{n}, x_{n+1}, x_{n+1}\right) \leq \alpha^{n} G\left(x_{n-1}, x_{n}, x_{n}\right)+\varphi^{n} G\left(x_{n}, x_{n+1}, x_{n+1}\right)
$$

and

$$
G\left(x_{n}, x_{n+1}, x_{n+1}\right) \leq \frac{\alpha^{n}}{1-\varphi^{n}} G\left(x_{n-1}, x_{n}, x_{n}\right)
$$

If we let $k^{n}=\frac{\alpha^{n}}{1-\varphi^{n}}$ For all $n \in \mathbb{N}$ where $0<k^{n}<1$ we have

$$
\left.\leq k^{n} G\left(x_{0}, x_{1}, x_{1}\right) \quad x_{n+1}, x_{n+1}\right) \leq k^{n} G\left(x_{n-1}, x_{n}, x_{n}\right)
$$

For all $n, m \in \mathbb{N}, m>n$, we have

$$
\begin{gathered}
G\left(x_{n}, x_{m}, x_{m}\right) \leq G\left(x_{n}, x_{n+1}, x_{n+1}\right)+G\left(x_{n+1}, x_{n+2}, x_{n+2}\right)+\cdots+G\left(x_{m-1}, x_{m}, x_{m}\right) \\
\leq k^{n} G\left(x_{0}, x_{1}, x_{1}\right)+k^{n+1} G\left(x_{0}, x_{1}, x_{1}\right)+\cdots+k^{m-1} G\left(x_{0}, x_{1}, x_{1}\right) \\
\leq\left(k^{n}+k^{n+1}+\cdots+k^{m-1}\right) G\left(x_{0}, x_{1}, x_{1}\right)
\end{gathered}
$$

Applying sum of geometric progression we have

$$
G\left(x_{n}, x_{n+1}, x_{n+1}\right) \leq \frac{k^{n}}{1-k} G\left(x_{0}, x_{1}, x_{1}\right)
$$

$\lim G\left(x_{n}, x_{m}, x_{m}\right)=0$ as $n, m \rightarrow \infty$. Therefore the sequence $\left\{x_{n}\right)$ is G-Cauchy sequence.

To show the uniqueness, suppose $p \neq q$ such that $T q=q$. Then

$$
G(p, q, q) \leq \alpha G(p, T p, T p)+\varphi G(p, q, q)
$$

This implies $p=q$.

Theorem 3.2: Let $(X, G)$ be a complete G-metric space, and let $T: X \rightarrow X$ be a mapping satisfying (3.11) for which there exist a monotonically decreasing function $a, b, c$ from $(0, \infty)$ into $[0,1]$ satisfying $a(t)+b(t)+c(t)<1$ such that, for each $x, y \in X ; x \neq y$.

$G(T x, T y, T y) \leq a G(x, y, y) G(x, T x, T x)+b G(x, y, y) G(y, T y, T y)+c G(x, y, y) G(x, y, y)$

Equivalently,

$G(T x, T y, T y) \leq a G(x, x, y) G(x, x, T x)+b G(x, x, y) G(y, y, T y)+c G(x, x, y) G(x, x, y)$

Then, there exists a unique fixed point $p$ in $T: T p=p$. 
Proof: For all $x_{0} \in X$ and Picard iteration $x_{n}=T^{n} x_{0}$ (3.11) or (3.12) we have

$$
\begin{gathered}
G\left(x_{n}, x_{n+1}, x_{n+1}\right)=G\left(T x_{n-1}, T x_{n}, T x_{n}\right) \\
\leq a G\left(x_{n-1}, x_{n}, x_{n}\right) G\left(x_{n-1}, x_{n}, x_{n}\right)+b G\left(x_{n-1}, x_{n}, x_{n}\right) G\left(x_{n}, x_{n+1}, x_{n+1}\right)+c G\left(x_{n-1}, x_{n}, x_{n}\right) G\left(x_{n-1}, x_{n}, x_{n}\right) \\
=a G\left(T x_{n-2}, T x_{n-1}, T x_{n-1}\right) G\left(T x_{n-2}, T x_{n-1}, T x_{n-1}\right)+b G\left(T x_{n-2}, T x_{n-1}, T x_{n-1}\right) G\left(T x_{n-1}, T x_{n}, T x_{n}\right) \\
+c G\left(T x_{n-2}, T x_{n-1}, T x_{n-1}\right) G\left(T x_{n-2}, T x_{n-1}, T x_{n-1}\right) \\
\leq a^{2} G\left(x_{n-2}, x_{n-1}, x_{n-1}\right) G\left(x_{n-2}, x_{n-1}, x_{n-1}\right)+b^{2} G\left(x_{n-2}, x_{n-1}, x_{n-1}\right) G\left(x_{n-1}, x_{n}, x_{n}\right) \\
+c^{2} G\left(x_{n-2}, x_{n-1}, x_{n-1}\right) G\left(x_{n-2}, x_{n-1}, x_{n-1}\right) \\
=a^{2} G\left(T x_{n-3}, T x_{n-2}, T x_{n-2}\right) G\left(T x_{n-3}, T x_{n-2}, T x_{n-2}\right)+b^{2} G\left(T x_{n-3}, T x_{n-2}, T x_{n-2}\right) G\left(T x_{n-2}, T x_{n-1}, T x_{n-1}\right) \\
+c^{2} G\left(T x_{n-3}, T x_{n-2}, T x_{n-2}\right) G\left(T x_{n-3}, T x_{n-2}, T x_{n-2}\right) \\
\leq a^{3} G\left(x_{n-3}, x_{n-2}, x_{n-2}\right) G\left(x_{n-3}, x_{n-2}, x_{n-2}\right)+b^{2} G\left(x_{n-3}, x_{n-2}, x_{n-2}\right) G\left(x_{n-2}, x_{n-1}, x_{n-1}\right) \\
+c^{2} G\left(x_{n-3}, x_{n-2}, x_{n-2}\right) G\left(x_{n-3}, x_{n-2}, x_{n-2}\right)
\end{gathered}
$$

Continue iteratively in this manner, then

$$
\begin{gathered}
G\left(x_{n}, x_{n+1}, x_{n+1}\right) \\
\leq a^{n} G\left(x_{0}, x_{1}, x_{1}\right) G\left(x_{0}, x_{1}, x_{1}\right)+b^{n} G\left(x_{0}, x_{1}, x_{1}\right) G\left(x_{1}, x_{2}, x_{2}\right)+c^{n} G\left(x_{0}, x_{1}, x_{1}\right) G\left(x_{0}, x_{1}, x_{1}\right) \\
\leq \frac{\left(a^{n}+c^{n}\right) G\left(x_{0}, x_{1}, x_{1}\right) G\left(x_{0}, x_{1}, x_{1}\right)}{1-b^{n} G\left(x_{1}, x_{2}, x_{2}\right)}
\end{gathered}
$$

By equating $j^{n}=a^{n}+c^{n}, g^{n}=1-b^{n}$ and $\alpha^{n}=\frac{j^{n}}{g^{n}}$. where $0 \leq \alpha^{n}<1$ We have

$G\left(x_{n}, x_{n+1}, x_{n+1}\right) \leq \alpha^{n} G\left(x_{0}, x_{1}, x_{1}\right)$

For all $n, m \in \mathbb{N}, m>n$, we have

$$
\begin{gathered}
G\left(x_{n}, x_{m}, x_{m}\right) \leq G\left(x_{n}, x_{n+1}, x_{n+1}\right)+G\left(x_{n+1}, x_{n+2}, x_{n+2}\right)+\cdots+G\left(x_{m-1}, x_{m}, x_{m}\right) \\
\leq \alpha^{n} G\left(x_{0}, x_{1}, x_{1}\right)+\alpha^{n+1} G\left(x_{0}, x_{1}, x_{1}\right)+\cdots+\alpha^{m-1} G\left(x_{0}, x_{1}, x_{1}\right) \\
\leq\left(\alpha^{n}+\alpha^{n+1}+\cdots+\alpha^{m-1}\right) G\left(x_{0}, x_{1}, x_{1}\right) \\
G\left(x_{n}, x_{n+1}, x_{n+1}\right) \leq \frac{\alpha^{n}}{1-\alpha} G\left(x_{0}, x_{1}, x_{1}\right)
\end{gathered}
$$

$\lim G\left(x_{n}, x_{m}, x_{m}\right)=0$ as $n, m \rightarrow \infty$. Therefore, the sequence $\left\{x_{n}\right)$ is G-Cauchy sequence.

To show the uniqueness, suppose $p \neq q$ such that $T q=q$. Then

$G(p, q, q) \leq a G(p, q, q) G(p, T p, T p)+b G(p, q, q) G(q, T q, T q)+c G(p, q, q) G(p, q, q)$

This implies $p=q$.

Theorem 3.4. Let $(X, G)$ be a complete G-metric space, and let $T: X \rightarrow X$ be a mapping satisfying (3.17) for which there exist nonnegative number $a, b, c$ satisfying $a+b+c<1$ such that, for each $x, y \in X$,

$G(T x, T y, T z) \leq a G(x, T x, T x)+b G(y, T y, T y)+c G(x, y, z)$

Equivalently

$G(T x, T y, T z) \leq a G(x, x, T x)+b G(y, y, T y)+c G(x, y, z)$

For all $x, y, z \in X$ where $0 \leq a+b+c<1$, then $T$ has a unique fixed point (say $p$, that is $T p=p$ ) and $T$ is $\mathrm{G}$ continuous at $p$.

Proof: Suppose that $T$ satisfies condition (3.17), then for all $x, y \in X$, we have

$$
\begin{gathered}
G(T x, T y, T y) \leq a G(x, T x, T x)+b G(y, T y, T y)+c G(x, y, y) \\
G(T x, T x, T y) \leq a G(x, x, T x)+b G(y, y, T y)+c G(x, x, y)
\end{gathered}
$$

Let $x_{n+1}=y_{n}$ and since $T$ is a contraction satisfying (3.11) or (3.12) we have

$$
\begin{gathered}
G\left(x_{n}, x_{n+1}, x_{n+1}\right)=G\left(T x_{n-1}, T x_{n}, T x_{n}\right) \\
\leq a G\left(x_{n-1}, x_{n}, x_{n}\right)+b G\left(x_{n}, x_{n+1}, x_{n+1}\right)+c G\left(x_{n}, x_{n+1}, x_{n+1}\right) \\
=a G\left(T x_{n-2}, T x_{n-1}, T x_{n-1}\right)+b G\left(T x_{n-1}, T x_{n}, T x_{n}\right)+c G\left(T x_{n-1}, T x_{n}, T x_{n}\right) \\
\leq a^{2} G\left(x_{n-2}, x_{n-1}, x_{n-1}\right)+b^{2} G\left(x_{n-1}, x_{n}, x_{n}\right)+c^{2} G\left(x_{n-1}, x_{n}, x_{n}\right) \\
=a^{2} G\left(T x_{n-3}, T x_{n-2}, T x_{n-2}\right)+b^{2} G\left(T x_{n-2}, T x_{n-1}, T x_{n-1}\right)+c^{2} G\left(T x_{n-2}, T x_{n-1}, T x_{n-1}\right) \\
\leq a^{3} G\left(x_{n-3}, x_{n-2}, x_{n-2}\right)+b^{3} G\left(x_{n-2}, x_{n-1}, x_{n-1}\right)+c^{3} G\left(x_{n-2}, x_{n-1}, x_{n-1}\right)
\end{gathered}
$$


Continue iteratively implies

$$
\begin{aligned}
& \leq \frac{a^{n}}{\left.1-x_{n}, x_{n+1}, x_{n+1}\right)} G\left(a^{n} G\left(x_{0}, x_{1}, x_{1}\right)+b^{n} G\left(x_{1}, x_{2}, x_{2}\right)+c^{n} G\left(x_{1}, x_{2}, x_{2}\right)\right. \\
& \text { let } \alpha^{n}=\frac{a^{n}-c^{n}}{1-b^{n}-c^{n}} \quad \text { where } 0 \leq \alpha^{n}<1
\end{aligned}
$$

Then

$G\left(x_{n}, x_{n+1}, x_{n+1}\right) \leq \alpha^{n} G\left(x_{0}, x_{1}, x_{1}\right)$

For all $n, m \in \mathbb{N}, m>n$

But

$G\left(x_{n}, x_{m}, x_{m}\right) \leq G\left(x_{n}, x_{n+1}, x_{n+1}\right)+G\left(x_{n+1}, x_{n+2}, x_{n+2}\right)+\cdots+G\left(x_{m-1}, x_{m}, x_{m}\right)$ $\leq \alpha^{n} G\left(x_{0}, x_{1}, x_{1}\right)+\alpha^{n+1} G\left(x_{0}, x_{1}, x_{1}\right)+\cdots+\alpha^{m-1} G\left(x_{0}, x_{1}, x_{1}\right)$ $\leq\left(\alpha^{n}+\alpha^{n+1}+\cdots+\alpha^{m-1}\right) G\left(x_{0}, x_{1}, x_{1}\right)$

$G\left(x_{n}, x_{n+1}, x_{n+1}\right) \leq \frac{\alpha^{n}}{1-\alpha} G\left(x_{0}, x_{1}, x_{1}\right)$

$\lim G\left(x_{n}, x_{m}, x_{m}\right)=0$ as $n, m \rightarrow \infty$. Therefore, the sequence $\left\{x_{n}\right)$ is G-Cauchy sequence.

To show the uniqueness, suppose $p \neq q$ such that $T q=q$. Then

$G(p, q, q) \leq a G(p, T p, T p)+b G(q, T q, T q)+c G(p, q, q)$

This implies $p=q$.

\section{CONCLUSION}

This work is an extension of Banach fixed point theorem to G-metric space. The existence and uniqueness of some fixed points under some weaker contractive conditions in complete G-metric space settings were obtained. The results are therefore refinements and generalizations of some recent results in the literature.

\section{REFERENCE}

Agarwal, R. P., Erdal Karapınar and Donal O'Regan., Fixed Point Theory in Metric Type Spaces, Springer Cham Heidelberg New York Dordrecht London.

Altun, I and Simsek, H., 2010. Some Fixed Point Theorems on Ordered Metric Spaces and Application, Hindawi Publishing Corporation Fixed Point Theory and Applications 2010, Article ID 621469,17 pages10.1155/2010/621469.

Banach, S., 1922. Sur les operations dans les ensembles et leur applications aux equations integrals. Fund. Math.3, 133-181.

Giniswamy, G and Maheshwari, P. G., 2014. Some Common Fixed Point Theorems on G-Metric Space, Gen. Math. Notes, 21 (2).

Meenakshi Gugnani., 2012. Madhu Agarwal., and Renu Chugh, Common Fixed Point Results in GMetric Spaces and Applications, International Journal of Computer Applications 43, (11): 0975 $-0987$.
Kumar, M., 2012. Compatible Maps in G-Metric Spaces, Int. Journal of Math. Analysis, 6, (29): 1415 1421.

Mustafa, $Z$ and Obiedat, H., 2010. A Fixed Point Theorem of Reich inG-Metric Spaces, CUBO A Mathematical Journal 12, (01): 83-93.

Mustafa, Z and Brailey Sims., 2006. A new approach to generalized metric spaces, Journal of Nonlinear and Convex Analysis 7, (2): 289-297.

Mustafa, Z., Obiedat, $\mathrm{H}$ and Awawdeh, F., 2008. Some Fixed Point Theorem for Mapping on Complete G-Metric Spaces, Hindawi Publishing Corporation Fixed Point Theory and Applications 2008, Article ID 189870, 12 pages doi:10.1155/2008/189870.

Rauf, K., Wahab, O. T and Ali, A., 2017. New Implicit Kirk-Type Schemes for General Class of QuasiContractive Operators in Generalized Convex Metric Spaces, The Australian, 2017 Journal of Mathematical Analysis and Applications(AJMAA): 14, (1): Article 8, 1-29.

Rhoade, B. E., 1977. A Comparison of Various Definitions of Contractive Mappings, Transactions of the American Mathematical Society 224, 257-290.

Singh, D., 2014. Some Best Proximity Point Theorems in G-Metric Spaces, National Institute of Technical Teachers' Training \& Research, Bhopal Under the Ministry of HRD, Govt. of India, October 30. 
Ughade, M and Daheriya, R. D., 2015. Tripled Fixed Point Results in Generalized Metric Spaces Under Nonlinear Type Contractions Depended on Another Function, Gazi University Journal of Science GU J. Sci. 28(1), 75 - 86.
Vats, R. K., Kumar, S and Sihag, V., 2013. Common Fixed Point Theorem for Expansive Mappings in G- Metric Spaces, Journal of mathematics and computer Science 6, 60-71. 